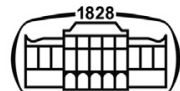

AKADÉMIAI KIADÓ

\section{Acta Veterinaria Hungarica}

68 (2020) 2, 123-129

DOI:

$10.1556 / 004.2020 .00019$

(c) 2020 Akadémiai Kiadó, Budapest

\section{ORIGINAL ARTICLE}

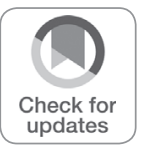

*Corresponding author. Tel.: +8254 912 0743; Fax: +82 54912 0759; E-mail: choys@korea.kr

\title{
Identification of B cell antigenome in Mycobacterium bovis by immunoproteomic analysis
}

\section{YUN SANG CHO* $\odot$, SANG EUN LEE, YOUNGBOO JANG, SUKCHAN JUNG and JONG MAN KIM}

Bacterial Disease Division, Department of Animal and Plant Health Research, Animal and Plant Quarantine Agency, Gimcheon-si, Gyeongsangbuk-do, Republic of Korea

Received: September 5, 2019 • Accepted: February 28, 2020

Published online: October 13, 2020

\section{ABSTRACT}

Bovine tuberculosis (bTB) is a common zoonosis prevalent in many countries with grave economic consequences. Most developed and developing countries have implemented the test-and-slaughter policy to protect public health and reduce economic losses in the cattle industry. The official diagnosis of bTB is based on assays dependent on cell-mediated immunity (CMI). CMI-based diagnosis demonstrates diagnostic incapability at late stages of infection, which could be overcome by diagnosis based on humoral immunity (HI). Therefore, there is an urgent need to identify and define the B cell antigenome of Mycobacterium bovis. In this study, the B cell antigenome of culture filtrate proteins (CFP) was defined by mass spectrometry-based proteomics technology. Four spots were detected on 2dimensional gel electrophoresis (2-DE) against M. bovis-positive serum in an immunoblotting experiment. Twenty-one proteins were identified in four spots by proteomic tools, such as Mb2900, Mb2898, Mb0448, Mb3834c, Mb1918c, Mb0134c, Mb0358 and Mb1868c, which are known B cell antigens, including 13 new proteins, i.e. Mb3751, Mb2006c, Mb3276c, Mb2244, Mb1164c, Mb2553c, Mb2946c, Mb1849c, Mb1511c, Mb1034c, Mb2616c, Mb0854c and Mb2267. These new proteins identified by 2$\mathrm{DE}$ and immunoblotting were the $\mathrm{B}$ cell antigens used in developing serological diagnostic methods based on HI to bTB.

\section{KEYWORDS}

B cell antigenome, Mycobacterium bovis, proteomic analysis, bovine tuberculosis, serological diagnosis, immunoblotting

\section{INTRODUCTION}

Bovine tuberculosis (bTB), a zoonosis caused by Mycobacterium bovis infection (Pesciaroli et al., 2014) in humans, has been reported in many countries (Cockle et al., 2002; Jeon et al., 2010; Bezos et al., 2014; Wanzala et al., 2017). In addition to economic losses due to slaughtering and compensation, outbreaks of bTB are a huge hurdle to international trade as importing countries demand bTB-free status in exporting countries (Trost et al., 2016). Therefore, most countries have implemented the test-and-slaughter policy for bTB.

Currently, the diagnosis of bTB is based on tests dependent on cell-mediated immunity (CMI), including the intradermal skin test (IST) and the $\gamma$-interferon assay using bovine purified protein derivative (bPPD) and other M. bovis-specific single antigens or antigen cocktails (Andersen et al., 2000; Infantes-Lorenzo et al., 2017). The diagnosis based on CMI is the best method in the early and middle stages of infection, but it cannot detect late-stage infection due to CMI status at that stage (de la Rua-Domenech et al., 2006). Therefore, cattle showing false negative results are highly dangerous and cannot be screened through CMI testing at late stages of infection. To expand the diagnostic window of bTB, diagnosis based on humoral immunity (HI) is needed as an ancillary method (de la Rua-Domenech et al., 2006; Bezos et al., 2014; 
Waters et al., 2015). Native proteins (P22, 20kDa, CMP and PPD) and recombinant proteins (MPB70, MPB83 and ESAT6) have been used as serological diagnostic antigens of bTB (Cho et al., 2007, 2009; Garbaccio et al., 2019; InfantesLorenzo et al., 2019; Souza et al., 2019). Recently, ELISA kits using Mb2900 (MPB70) and Mb2898 (MPB83) recombinant protein antigens have been commercialised. However, in order to further improve the sensitivity and specificity of the diagnosis, it is necessary to discover new antibody-specific protein antigens. Therefore, identification of $\mathrm{B}$ cell antigens in M. bovis is urgently needed for the development of HI-based diagnosis. In this study, the culture filtrate proteins (CFP) from $M$. bovis AN5, a standard strain used in bPPD production, were analysed by proteomic and immunological tools, such as 1-dimensional SDS-PAGE (1-DE), 2-dimensional gel electrophoresis (2-DE), western blotting, and liquid chromatography (LC)-based proteomic analysis.

The proteins from $M$. bovis AN5 cells and the culture supernatant were fractionated into CFP from the supernatant and soluble (SOL) and insoluble proteins (INS) from the cells. Each fraction was analysed by 1- and 2-DE. Consequently, M. bovis-specific proteins were examined by western blotting using $M$. bovis positive serum, and the immunoreactive spots of CFP on 2-DE were identified by ingel digestion and proteomic tools.

To our knowledge, this is the first study devoted to the identification of the $M$. bovis antigenome, which could provide insights into the development of serological diagnostics for the supplementary diagnosis of bTB.

\section{MATERIALS AND METHODS}

\section{Ethics statement}

Animal and Plant Quarantine Agency (APQA) is a national central research institute in the Republic of Korea that has been researching and developing diagnosis, treatment, and prevention technologies for animal diseases since 1911. The Institutional Animal Care and Use Committee (IAUUC) of APQA for animal testing was first organized and operated in 2008. This study was conducted prior to the organization of IAUUC in APQA, and the regulations on animal welfare were conducted in accordance with general regulations. Blood was collected at the time of bTB testing, and bTB-positive sample collection was performed at the time of slaughtering it in accordance with national animal disease prevention policy.

\section{Cell cultivation and sample preparation}

Mycobacterium bovis AN5 was used for the production of CFP, which had been used for the production of purified protein derivative (PPD) tuberculin at the Animal and Plant Quarantine Agency in the Republic of Korea (Cho et al., 2015). Mycobacterium bovis AN5 was cultured in Sauton broth (asparagine $4.8 \mathrm{~g}$, citric acid $2.4 \mathrm{~g}, \mathrm{MgSO}_{4} 0.6 \mathrm{~g}, \mathrm{~K}_{2} \mathrm{HPO}_{4}$ $0.6 \mathrm{~g}$, ferric ammonium citrate $0.06 \mathrm{~g}$, glycerine $72 \mathrm{~mL}, \mathrm{ZnSO}_{4}$ $0.0096 \mathrm{~g}, \mathrm{CuSO}_{4} 0.0012 \mathrm{~g}$, ammonia water $2.7 \mathrm{~mL}$, distilled water $1,200 \mathrm{~mL}, \mathrm{pH} 7.0-7.2$ ) for 8 weeks at $37^{\circ} \mathrm{C}$. For the preparation of CFP, cultures were sterilised by heating for $3 \mathrm{~h}$ at $100{ }^{\circ} \mathrm{C}$. Sterilised cultures were ultracentrifuged at $20,000 \times g$ for 30 min using a Beckman L8-M ultracentrifuge (Beckman Coulter, Inc., Atlanta, Georgia, USA). The supernatant was precipitated by adding $85 \%(\mathrm{w} / \mathrm{v})$ ammonium sulphate (Sigma Chemical, St. Louis, Missouri, USA). The precipitated supernatant was centrifuged at $10,000 \times g$ for $10 \mathrm{~min}$, and the precipitate was transferred to a dialysis tube (molecular weight cut-off 3,500 Da) (Sigma Chemical, St. Louis, Missouri, USA). The precipitant was dialysed for $18 \mathrm{~h}$ against $0.01 \mathrm{M}$ phosphate buffered saline, filtered by a $0.45-\mu \mathrm{m}$ filter and the protein concentration calculated using the BCA protein assay kit (Pierce Biotechnology, Rockford, IL, USA). All examined proteins were used at a concentration of $40 \mu \mathrm{g} / \mathrm{mL}$.

For the preparation of the SOL and INS fractions, the cells were harvested by centrifugation at $2,500 \times g$ for $15 \mathrm{~min}$ after heating $M$. bovis cells. Harvested cells were sonicated and centrifuged to remove non-lysed cells and cellular debris at $5,000 \times g$ for $10 \mathrm{~min}$. The supernatant was ultracentrifuged at $27,000 \times g$ for $10 \mathrm{~min}$ to remove cell debris and the supernatant was then ultracentrifuged at $100,000 \times g$ for $2 \mathrm{~h}$. The supernatant was used as the SOL fraction and the pellet as the INS fraction.

\section{Electrophoresis and immunoblotting}

One-DE was performed using a standard protocol (Cho et al., 2015). Two-DE for CFP was performed using an immobilised $\mathrm{pH}$ gradient (IPG strip; $\mathrm{pH} 4-7$ ) for $1-\mathrm{DE}$ and $12.5 \%$ polyacrylamide gel for 2-DE (Mollenkopf et al., 1999). For western blotting, 2-DE was performed using a Zoom IPG runner strip ( $\mathrm{pH} 4-7$; Invitrogen Co., Carlsbad, CA, USA) for 1-DE and 4-12\% Bis-Tris NuPAGE gel (Invitrogen Co., Carlsbad, CA, USA) for 2-DE. One gel was stained with Coomassie blue for in-gel digestion and another gel was stained with silver nitrate (Sigma Chemical, St. Louis, Missouri, USA) for protein profiling. Another gel was used for western blotting with $M$. bovis-positive serum, which was obtained from cattle immunised with M. bovis AN5 (Cho et al., 2009). The immunoreactive protein spots on the $2-\mathrm{DE}$ gel were examined visually. The first gel to show a protein pattern was destained with $50 \%$ methanol for in-gel extraction of immunoreactive spots numbered 1 to 4 , for proteomic analysis by nanoscale LC coupled to tandem mass spectrometry (nano LC-MS/MS) (Thermo Electron, San Jose, CA, USA), in order to define the $B$ cell antigenome of CFP.

\section{In-gel digestion of 2-DE spots and B cell antigenome analysis}

Each spot detected in western blotting was excised from the gel stained with Coomassie blue. After in-gel digestion with trypsin (Roche, Basle, Switzerland), peptides obtained from the spots were analysed by an Agilent 1100 Series Nano-LC and LTQmass spectrometer (Thermo Electron, San Jose, CA, USA). Proteins were identified by peptide mass fingerprinting (PMF) using MASCOT search engine (score: >100) and BLAST analysis, in reference to $M$. bovis protein databases [National 
Center for Biotechnology Information (NCBI) GenBank accession No. BX248333, 3920 entries] (Sartain et al., 2006).

\section{RESULTS}

\section{Protein profiling by 1- and 2-DE}

The major proteins identified from 1-DE profiling of CFP were different from those of SOL and INS (Fig. 1A). The major antigens identified on 1-DE of CFP were 15, 20 and $25 \mathrm{kDa}$ in size, while those in SOL were 15,25 , and $50 \mathrm{kDa}$, and those in INS were 25 and $35 \mathrm{kDa}$ in size. By the protein profile on 2-DE analysis of samples from $M$. bovis AN5 CFP, most proteins were acidic (pI 4-5.5) and below $75 \mathrm{kDa}$ in size (Fig. 2A).

\section{Mycobacterium bovis-specific antigens observed on 1-DE}

The antigenic protein of CFP was $20 \mathrm{kDa}$ in size as observed on 1-DE and western blotting with the M. bovis-positive serum, obtained by immunisation with formalinised $M$. bovis AN5. However, antigens of CFP specific to bTB ISTpositive reactor serum were 20,60 and $80 \mathrm{kDa}$ in size (Fig. 1B and C). The specific antigenic pattern of CFP differed depending on the source of serum, one of which was obtained by immunisation with $M$. bovis AN5 formalinised bacterin (Fig. 1B) and the other was from a bPPD-positive reactor in a cattle farm (Fig. 1C). This implies that different antigen-antibody reactions occur in different stages of infection probably due to the difference in immune status. INS proteins were confirmed by western blotting to be of size $50 \mathrm{kDa}$ (Fig. $1 \mathrm{~B}$ and $\mathrm{C}$ ).

\section{Identification of $M$. bovis-specific antigens on 2-DE}

Specific reactive antigen groups on 2-DE showed 4 spots, all of which were acidic (pI 4.3-5.5) and less than $75 \mathrm{kDa}$ in size. Molecular weight (MW, kDa)/pI of the 4 spots were 22/ 4.1, 60/4.7, 50/4.2 and 70/4.7, respectively (Fig. 2A). The immunoreactivity of spot 1 was the highest among the 4 spots, with the other 3 spots being very weak (Fig. 2B). Twenty-one proteins identified from the $4 \mathrm{M}$. bovis-specific spots were Mb2900 (MPB70), Mb2898 (MPB83), Mb3751 (Cut5), Mb0448 (GroEL2), Mb2006c (Cfp21), Mb3276c (SahH), Mb2244 (GlnA1), Mb1164c (MetE), Mb3834c (FbpA), Mb1918c (FbpB), Mb0134c (FbpC), Mb2553c (Fas), Mb2946c (Smc), Mb1849c (PE_PGRS33), Mb0358 (DnaK), Mb1511c (Acn), Mb2616c, Mb0854c, Mb2267 (FabD), Mb1868c (GlcB) and Mb1034c (MetS) (Table 1). Of these identified antigens, Mb2900 (MPB70) had been previously used to detect M. bovis-specific antibody (Cho et al., 2007). Mb2898 (MPB83) and Mb2002c (MPB64) were the other well-known B cell antigens confirmed in this study (Table 1) (Andersen et al., 1991; McNair et al., 2001). Western blotting on 2-DE allowed the identification of 13 new proteins as B cell antigen candidates, which were Mb3751 (Cut5), Mb2006c (Cfp21), Mb3276c (SahH), Mb2244 (GlnA1), Mb1164c (MetE), Mb2553c (Fas), Mb2946c (Smc),
A

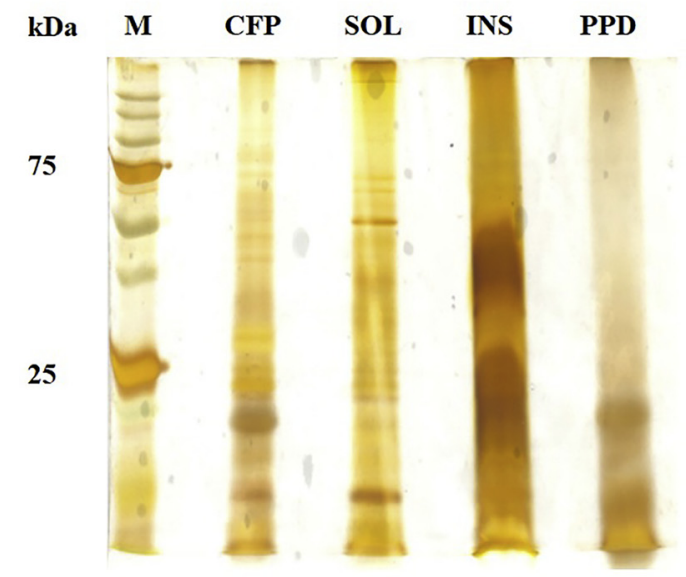

B

PPD CFP INS SOL

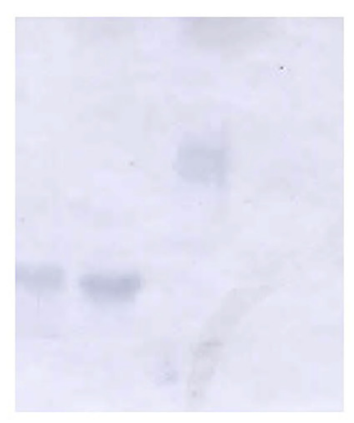

C

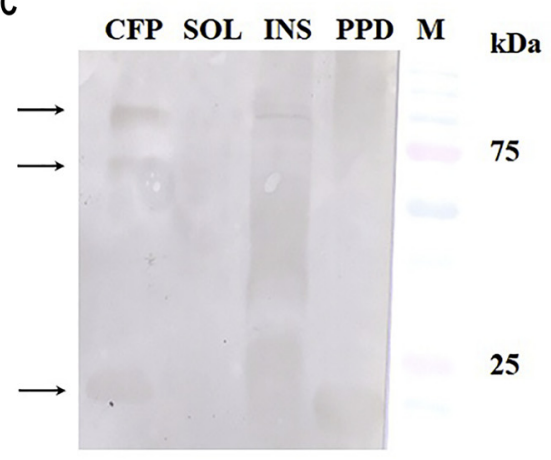

Fig. 1. One dimensional SDS-PAGE and immunoblotting. (A) Silver staining of each fraction from Mycobacterium bovis; (B) Western blotting with serum from $M$. bovis AN5-immunised cattle; (C) Western blotting with serum from bTB IST positive cattle. M Molecular weight marker (BioRad Co.); CFP - culture filtrate protein; SOL - soluble protein; INS - insoluble protein; PPD purified protein derivative

Mb1849c (PE_PGRS33), Mb1511c (Acn), Mb1034c (MetS), Mb2616c, Mb0854c and Mb2267 (FabD).

\section{DISCUSSION}

Four major protein spots from $M$. bovis AN5 CFP reacted with $M$. bovis AN5 antiserum-identified proteins with the following MW (kDa)/pI parameters: 20/4.3 (spot 1), 60/4.8 

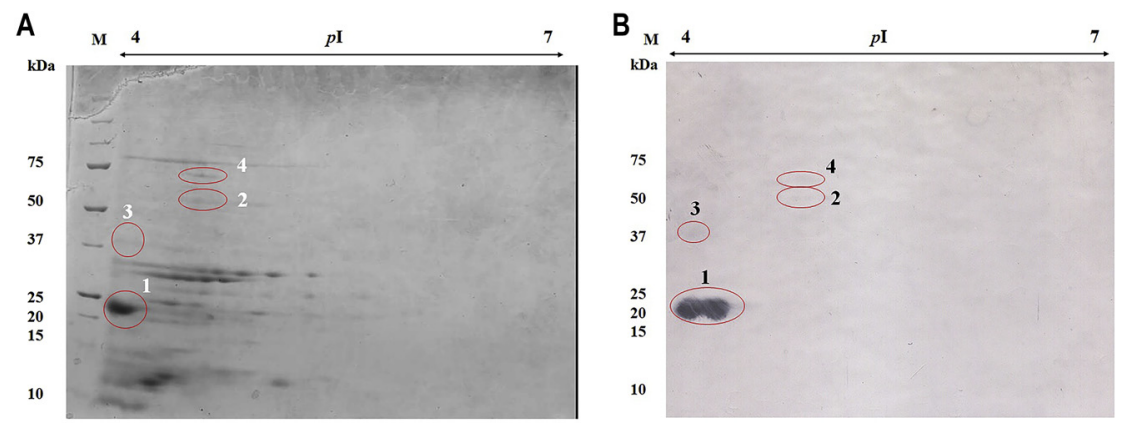

Fig. 2. Profile of 2-dimensional electrophoresis (A) and immunoblotting (B) of CFP. (A) Major proteins were found distributed within a pI range of 4-6 and a molecular weight of $10-75 \mathrm{kDa}$. (B) Four spots were reacted with serum from M. bovis AN5-immunised cattle. The predominant immunoreactive spot was in the group with a molecular weight of $20 \mathrm{kDa}$ and $\mathrm{pI} 4$

(spot 2), 50/4/3 (spot 3) and 70/4.8 (spot 4). The proteins from the 4 spots were extracted, trypsinised and analysed by nano LC-MS/MS. The B cell antigenome of CFP of M. bovis was found to consist of 21 proteins, which were Mb2900, Mb2898, Mb3751, Mb0448, Mb2006c, Mb3276c, Mb2244, Mb1164c, Mb3834c, Mb1918c, Mb0134c, Mb2553c, Mb2946c, Mb1849c, Mb0358, Mb1511c, Mb2616c, Mb0854c, Mb2267, Mb1868c and Mb1034c. The 4 proteins from the highest reactive spot 1 were Mb2900, Mb3751, $\mathrm{Mb2006c}$ and Mb2898, which would be useful for developing antibody-based diagnostic methods such as ELISA (Waters et al., 2015). The optimal combination of specific antigens will be useful in developing B cell antigen cocktails for HI-based diagnosis of bTB (Amadori et al., 2002).

Protein profiles from different fractions obtained from M. bovis AN5 were different. Proteins detected on 1-DE of CFP were different from those detected in the INS and SOL fractions (Fig. 1A). PPD was produced by a similar method as CFP with the exception of the precipitation method and

Table 1. Proteins identified through B cell antigenomic analysis of culture filtrate protein from Mycobacterium bovis AN5

\begin{tabular}{|c|c|c|c|c|c|c|c|c|}
\hline $\begin{array}{l}\text { Spot } \\
\text { number }\end{array}$ & $\begin{array}{r}\text { Theoretical MW } \\
(\mathrm{kDa}) / \mathrm{pI}\end{array}$ & Score & $\begin{array}{r}\text { No. of } \\
\text { peptides }\end{array}$ & $\begin{array}{r}\mathrm{MW}(\mathrm{kDa}) / \mathrm{pI} \text { on } \\
2-\mathrm{DE}\end{array}$ & $\begin{array}{l}\text { Protein } \\
\text { name }\end{array}$ & $\begin{array}{c}\mathrm{Mb} \\
\text { number }\end{array}$ & $\begin{array}{c}\mathrm{Rv} \\
\text { number }\end{array}$ & $\begin{array}{c}\text { B cell } \\
\text { antigenicity }\end{array}$ \\
\hline \multirow[t]{4}{*}{1} & $19 / 4.5$ & 248 & 4 & $22 / 4.1$ & MPB70 & Mb2900 & Rv2875 & B \\
\hline & $23 / 6.1$ & 170 & 3 & $22 / 4.1$ & Cut5 & Mb3751 & Rv3724A & - \\
\hline & $22 / 5.9$ & 162 & 4 & $22 / 4.1$ & Cfp21 & Mb2006c & Rv1984c & - \\
\hline & $22 / 4.7$ & 148 & 4 & $22 / 4.1$ & MPB83 & Mb2898 & Rv2873 & B \\
\hline \multirow[t]{5}{*}{2} & $57 / 4.6$ & 426 & 10 & $60 / 4.7$ & GroEL2 & Mb0448 & Rv0440 & B \\
\hline & $54 / 4.9$ & 240 & 6 & $60 / 4.7$ & SahH & Mb3276c & Rv3248c & - \\
\hline & $54 / 4.8$ & 140 & 4 & $60 / 4.7$ & $\mathrm{Gln} A 1$ & Mb2244 & Rv2220 & - \\
\hline & $82 / 4.9$ & 134 & 9 & $60 / 4.7$ & MetE & Mb1164c & Rv1133c & - \\
\hline & $36 / 6.5$ & 108 & 1 & $60 / 4.7$ & $\mathrm{FbpA}$ & Mb3834c & $\operatorname{Rv} 3804 c$ & B \\
\hline \multirow[t]{3}{*}{3} & $326 / 4.8$ & 112 & 13 & $50 / 4.2$ & Fas & Mb2553c & Rv2524c & - \\
\hline & $131 / 4.9$ & 106 & 4 & $50 / 4.2$ & $\mathrm{Smc}$ & Mb2946c & Rv2922c & - \\
\hline & $41 / 3.9$ & 101 & 7 & $50 / 4.2$ & PE_PGRS33 & Mb1849c & Rv1818c & - \\
\hline \multirow[t]{13}{*}{4} & $82 / 4.9$ & 598 & 17 & $70 / 4.7$ & MetE & Mb1164c & Rv1133c & - \\
\hline & $36 / 6.5$ & 459 & 10 & $70 / 4.7$ & FbpA & Mb3834c & Rv3804c & B \\
\hline & $67 / 4.6$ & 450 & 14 & $70 / 4.7$ & DnaK & Mb0358 & Rv0350 & B \\
\hline & $36 / 6.5$ & 386 & 9 & $70 / 4.7$ & FbpA & Mb3834c & Rv3804c & B \\
\hline & $37 / 6.3$ & 345 & 9 & $70 / 4.7$ & $\mathrm{FbpC}$ & $\mathrm{Mb} 0134 \mathrm{c}$ & Rv0129c & B \\
\hline & $102 / 4.7$ & 321 & 11 & $70 / 4.7$ & Acn & Mb1511c & Rv1475c & - \\
\hline & $58 / 5.6$ & 307 & 7 & $70 / 4.7$ & MetS & $\mathrm{Mb} 1034 \mathrm{c}$ & Rv1007c & - \\
\hline & $59 / 5.7$ & 302 & 6 & $70 / 4.7$ & Mb2616c & Mb2616c & $\operatorname{Rv} 2585 c$ & - \\
\hline & $35 / 5.7$ & 278 & 6 & $70 / 4.7$ & FbpB & Mb1918c & Rv1886c & B \\
\hline & $30 / 4.9$ & 237 & 6 & $70 / 4.7$ & Mb0854c & Mb0854c & Rv0831c & - \\
\hline & $31 / 4.6$ & 182 & 14 & $70 / 4.7$ & FabD & Mb2267 & Rv2243 & - \\
\hline & $80 / 4.8$ & 182 & 5 & $70 / 4.8$ & GlcB & Mb1868c & Rv1837c & B \\
\hline & $57 / 4.6$ & 147 & 4 & $70 / 4.8$ & GroEL2 & Mb0448 & Rv0440 & B \\
\hline
\end{tabular}

MW $=$ molecular weight.

Data from identification and characterisation (http://genolist.pasteur.fr/BoviList/), B cell epitope (http://immuneepitope.org/home.do). Localisation (http://www.iedb.org/). 
an additional washing step to discard the lipid component and other contaminants (Cho et al., 2015). Conventionally, PPD and CFP have been used as native ELISA antigens for bTB serological diagnosis (Liu et al., 2007; Jeon et al., 2010; Souza et al., 2012). The classical ELISA antigen observed on $1-\mathrm{DE}$ was mainly a $20-\mathrm{kDa}$ protein, and was considered to be Mb2900 (MPB70). As shown in Fig. 1A, CFP shows more protein bands compared to PPD. Therefore, CFP might be more optimal for use as a bTB ELISA antigen due to better coverage towards different antibodies produced by different immunoreactivity to $M$. bovis infection. We observed that different sources of serum showed different antibody-specific antigen patterns (Fig. $1 \mathrm{~A}$ and $\mathrm{B}$ ). This implies that antigen cocktails containing more proteins will detect a broader range of serum antibodies. In this study, $M$. bovisspecific antibody samples were very limited $(n=2)$. It is anticipated that the major antigen reacting with antibody produced by $M$. bovis infection will be around $20 \mathrm{kDa}$ in size and would be mainly composed of Mb2900. An antigen mixture will be able to detect more antibodies and hence amplify the distinction between an infected and uninfected individual, thereby improving diagnostic accuracy and sensitivity. The sensitivity of the antigen mixture should be evaluated by testing against $M$. bovis-negative antibodies. Meanwhile, the protein profiles of SOL and INS were different from those of CFP and PPD (Fig. 1A). In addition, the immunoreactive antigen patterns from SOL and INS were different compared with that of CFP. This implies that bTB ELISA using CFP antigens is superior, compared with other antigens obtained from SOL and INS.

In 2-DE analysis of CFP, 20-kDa Mb2900 (MPB70) proteins from spot 1 were the major antigenic proteins (Fig. 2A). This implies that traditional bTB ELISA antigens based on Mb2900 (MPB70) are effective antigens for bTB serological diagnosis. Interestingly, the three proteins from spot 1 were shared with other ELISA antigens Mb2900, Mb2006c, and Mb2898 (Cho et al., 2009) and reacted with the M. bovis antibody. The reaction of these three spots was very weak, and therefore inconclusive (Fig. 2B). However, the serological diagnostic sensitivity of MPB70-based ELISA could be improved by adding these weakly reacting protein groups. Meanwhile, as shown in Fig. 2, other non-reactive proteins in CFP could lead to non-specific immune reactions in the antibody detection of $M$. bovis-free cattle. Therefore, CFP antigens in bTB ELISA have shown more non-specific reactions than the fractionated protein antigens (Cho et al., 2007, 2009). In the future, the recombinant protein cocktails composed of $B$ cell antigenome or fractionated proteins will be more sensitive and specific in the serological diagnosis of bTB.

In 1-DE immunoblotting with different sources of serum, the reactive protein antigens of SOL, INS and CFP were different (Fig. 1B and C). We used two kinds of positive sera, of which one was from naturally infected cattle showing positive reaction in IST, and another one was from cattle immunised with $M$. bovis AN5 bacterin. Each serum originated from different $M$. bovis, the reference AN5 strain and the field strain. M. bovis AN5 has been used for PPD tuberculin production worldwide, like in the Republic of Korea, and has been adapted for use in artificial culture medium (Cho et al., 2015). The protein profiles obtained from each strain on 2-DE were different (data not shown). Therefore, antisera from cattle infected with different antigens are different, and immunoreactivity to the same protein is different according to the different sources of serum (Fig. 1B and C). Theoretically, some 3,953 proteins could be expressed from the M. bovis genome (Cho et al., 2015). Therefore, B cell antigenome from different sources of $M$. bovis infection including various field isolates should be investigated to improve the sensitivity and specificity of bTB serological diagnosis. In order to achieve this, B cell antigenic cocktails would be preferred over the use of single proteins, for sensitive antibody detection of bTB (Liu et al., 2007).

Twenty-one proteins identified in the B cell antigenome in CFP include 8 known B cell antigens, Mb2900, Mb2898, Mb0448, Mb3834c, Mb0358, Mb0134c, Mb1918c, and Mb1868c (Table 1). Mb2900 (MPB70) has been used as a bTB ELISA antigen to detect $M$. bovis-specific antibodies for a long time (Cho et al., 2007). Mb2898 (MPB83) and Mb2002c (MPB64), the other well-known B cell antigens like Mb2900 (MPB70), were also confirmed in this study (Table 1) (Andersen et al., 1991; McNair et al., 2001). In a previous study involving the identification of CFP in Mycobacterium tuberculosis, Rv1636 (TB15.3), Rv2031c (HspX), Rv3740, Rv2524c (Fas), Rv1037c (EsxI) and Rv0138 have been identified (Meikle et al., 2009). However, the previous experiments did not include western blotting. In this study, the B cell antigenome of M. bovis CFP was identified by western blotting after 2-DE (Fig. 2B, Table 1 and Supplementary files). The serological assay used in the diagnosis of human TB has involved the use of Rv3875 (ESAT6), Rv3874 (CFP10), Rv1886c (Ag85B), Rv2031c (Hsp16.3) and the fusion protein Ag85B-Hsp16.3 as antigens (Zhang et al., 2015). Furthermore, this serological diagnosis can differentiate between active and latent TB infection (Zhang et al., 2015). In bTB antibody detection, an IDEXX commercial kit for bTB serological diagnosis has been used with Mb2900 (MBP70) and Mb2898 (MPB83) as recombinant antigens (Waters et al., 2015). The other B cell antigens, identified in this study, will be very useful as candidates in bTB antigen cocktails. Interestingly, out of the B cell antigens identified in this study, 13 were novel proteins, Mb3751 (Cut5), Mb2006c (Cfp21), Mb3276c (SahH), Mb2244 (GlnA1), Mb1164c (MetE), Mb2553c (Fas), Mb2946c (Smc), Mb1849c (PE_PGRS33), Mb1511c (Acn), Mb1034c (MetS), Mb2616c, Mb0854c and Mb2267 (FabD). Therefore, the newly identified antigens could be candidates in $M$. bovis antibody detection to improve the sensitivity of current bTB diagnosis (Souza et al., 2012; Casal et al., 2014). The potential antigens identified in this study should be confirmed for antibody reaction with the pure native and/or recombinant form of the antigen.

\section{ACKNOWLEDGEMENTS}

This study was supported by the Animal and Plant Quarantine Agency, Republic of Korea. We thank Dr Mun Han Lee (Seoul National University) and John T. Belisle 
(Colorado State University) for their critical advice in completing this study. We also thank Yonsei Proteome Research Center for the proteomic analysis.

\section{REFERENCES}

Amadori, M., Lyashchenko, K. P., Gennaro, M. L., Pollock, J. M. and Zerbini, I. (2002): Use of recombinant proteins in antibody tests for bovine tuberculosis. Vet. Microbiol. 85, 379-389.

Andersen, A. B., Ljungqvist, L., Haslov, K. and Bentzon, M. W. (1991): MPB 64 possesses 'tuberculosis-complex'-specific Band T-cell epitopes. Scand. J. Immunol. 34, 365-372.

Andersen, P., Munk, M. E., Pollock, J. M. and Doherty, T. M. (2000): Specific immune-based diagnosis of tuberculosis. Lancet 356, 1099-1104.

Bezos, J., Casal, C., Romero, B., Schroeder, B., Hardegger, R., Raeber, A. J., Lopez, L., Rueda, P. and Dominguez, L. (2014): Current ante-mortem techniques for diagnosis of bovine tuberculosis. Res. Vet. Sci. 97 Suppl., S44-S52.

Casal, C., Diez-Guerrier, A., Alvarez, J., Rodriguez-Campos, S., Mateos, A., Linscott, R., Martel, E., Lawrence, J. C., Whelan, C., Clarke, J., O'Brien, A., Dominguez, L. and Aranaz, A. (2014): Strategic use of serology for the diagnosis of bovine tuberculosis after intradermal skin testing. Vet. Microbiol. 170, 342-351.

Cho, Y. S., Jang, Y. B., Lee, S. E., Cho, J. Y., Ahn, J. M., Hwang, I., Heo, E., Nam, H. M., Cho, D., Her, M., Jean, Y. H., Jung, S. C., Kim, J. M., Lee, H. S., Lee, K. and Belisle, J. T. (2015): Short communication: proteomic characterization of tuberculin purified protein derivative from Mycobacterium bovis. Res. Vet. Sci. 101, 117-119.

Cho, Y. S., Jung, S. C., Kim, J. M. and Yoo, H. S. (2007): Enzyme-linked immunosorbent assay of bovine tuberculosis by crude mycobacterial protein 70. J. Immunoassay Immunochem. 28, 409-418.

Cho, Y. S., Lee, S. E., Ko, Y. J., Cho, D., Lee, H. S., Hwang, I., Nam, H., Heo, E., Kim, J. M. and Jung, S. (2009): Definition of purified enzyme-linked immunosorbent assay antigens from the culture filtrate protein of Mycobacterium bovis by proteomic analysis. J. Immunoassay Immunochem. 30, 291-304.

Cockle, P. J., Gordon, S. V., Lalvani, A., Buddle, B. M., Hewinson, R. G. and Vordermeier, H. M. (2002): Identification of novel Mycobacterium tuberculosis antigens with potential as diagnostic reagents or subunit vaccine candidates by comparative genomics. Infect. Immun. 70, 6996-7003.

de la Rua-Domenech, R., Goodchild, A. T., Vordermeier, H. M., Hewinson, R. G., Christiansen, K. H. and Clifton-Hadley, R. S. (2006): Ante mortem diagnosis of tuberculosis in cattle: a review of the tuberculin tests, gamma-interferon assay and other ancillary diagnostic techniques. Res. Vet. Sci. 81, 190-210.

Garbaccio, S. G., Garro, C. J., Delgado, F., Tejada, G. A., Eirin, M. E., Huertas, P. S., Leon, E. A. and Zumarraga, M. J. (2019): Enzyme-linked immunosorbent assay as complement of intradermal skin test for the detection of Mycobacterium bovis infection in cattle. Tuberculosis (Edinb.) 117, 56-61.

Infantes-Lorenzo, J. A., Moreno, I., Risalde, M. L. A., Roy, A., Villar, M., Romero, B., Ibarrola, N., de la Fuente, J., Puentes, E., de Juan, L., Gortazar, C., Bezos, J., Dominguez, L. and Dominguez, M. (2017): Proteomic characterisation of bovine and avian purified protein derivatives and identification of specific antigens for serodiagnosis of bovine tuberculosis. Clin. Proteomics 14, 36.

Infantes-Lorenzo, J. A., Moreno, I., Roy, A., Risalde, M. A., Balseiro, A., de Juan, L., Romero, B., Bezos, J., Puentes, E., Akerstedt, J., Tessema, G. T., Gortazar, C., Dominguez, L. and Dominguez, M. (2019): Specificity of serological test for detection of tuberculosis in cattle, goats, sheep and pigs under different epidemiological situations. BMC Vet. Res. 15, 70.

Jeon, B. Y., Kim, S. C., Je, S., Kwak, J., Cho, J. E., Woo, J. T., Seo, S., Shim, H. S., Park, B. O., Lee, S. S. and Cho, S. N. (2010): Evaluation of enzyme-linked immunosorbent assay using milk samples as a potential screening test of bovine tuberculosis of dairy cows in Korea. Res. Vet. Sci. 88, 390-393.

Liu, S., Guo, S., Wang, C., Shao, M., Zhang, X., Guo, Y. and Gong, Q. (2007): A novel fusion protein-based indirect enzyme-linked immunosorbent assay for the detection of bovine tuberculosis. Tuberculosis (Edinb.) 87, 212-217.

McNair, J., Corbett, D. M., Girvin, R. M., Mackie, D. P. and Pollock, J. M. (2001): Characterization of the early antibody response in bovine tuberculosis: MPB83 is an early target with diagnostic potential. Scand. J. Immunol. 53, 365-371.

Meikle, V., Alito, A., Llera, A. S., Gioffre, A., Peralta, A., Buddle, B. M., Cataldi, A. (2009): Identification of novel Mycobacterium bovis antigens by dissection of crude protein fractions. Clin. Vaccine Immunol. 16, 1352-1359.

Mollenkopf, H. J., Jungblut, P. R., Raupach, B., Mattow, J., Lamer, S., Zimny-Arndt, U., Schaible, U. E. and Kaufmann, S. H. (1999): A dynamic two-dimensional polyacrylamide gel electrophoresis database: the mycobacterial proteome via Internet. Electrophoresis 20, 2172-2180.

Pesciaroli, M., Alvarez, J., Boniotti, M. B., Cagiola, M., Di Marco, V., Marianelli, C., Pacciarini, M. and Pasquali, P. (2014): Tuberculosis in domestic animal species. Res. Vet. Sci. 97Suppl., S78-S85.

Sartain, M. J., Slayden, R. A., Singh, K. K., Laal, S. and Belisle, J. T. (2006): Disease state differentiation and identification of tuberculosis biomarkers via native antigen array profiling. Mol. Cell. Proteomics 5, 2102-2113.

Souza, I. I., Melo, E. S., Ramos, C. A., Farias, T. A., Osorio, A. L., Jorge, K. S., Vidal, C. E., Silva, A. S., Silva, M. R., Pellegrin, A. O. and Araujo, F. R. (2012): Screening of recombinant proteins as antigens in indirect ELISA for diagnosis of bovine tuberculosis. SpringerPlus 1, 77.

Souza, I. I. F., Rodrigues, R. A., Goncalves Jorge, K. S., Silva, M. R., Lilenbaum, W., Vidal, C. E. S., Etges, R. N., Kostovic, M. and Araujo, F. R. (2019): ELISA using a recombinant chimera of ESAT-6/MPB70/MPB83 for Mycobacterium bovis diagnosis in naturally infected cattle. J. Vet. Med. Sci. 81, 9-14.

Trost, B., Stuber, T., Surujballi, O., Nelson, J., Robbe-Austerman, S., Smith, N. H., Desautels, L., Tikoo, S. K. and Griebel, P. (2016): Investigation of the cause of geographic disparities in IDEXX ELISA sensitivity in serum samples from Mycobacterium bovisinfected cattle. Sci. Rep. 6, 22763.

Wanzala, S. I., Palmer, M. V., Waters, W. R., Thacker, T. C., Carstensen, M., Travis, D. A. and Sreevatsan, S. (2017): Evaluation of pathogen-specific biomarkers for the diagnosis of tuberculosis in white-tailed deer (Odocoileus virginianus). Am. J. Vet. Res. 78, 729-734. 
Waters, W. R., Palmer, M. V., Stafne, M. R., Bass, K. E., Maggioli, M. F., Thacker, T. C., Linscott, R., Lawrence, J. C., Nelson, J. T., Esfandiari, J., Greenwald, R. and Lyashchenko, K. P. (2015): Effects of serial skin testing with purified protein derivative on the level and quality of antibodies to complex and defined antigens in Mycobacterium bovis-infected cattle. Clin. Vaccine Immunol. 22, 641-649.

Zhang, C., Song, X., Zhao, Y., Zhang, H., Zhao, S., Mao, F., Bai, B., Wu, S. and Shi, C. (2015): Mycobacterium tuberculosis secreted proteins as potential biomarkers for the diagnosis of active tuberculosis and latent tuberculosis infection. J. Clin. Lab. Anal. 29, 375-382.

\section{SUPPLEMENTARY DATA}

Supplementary data to this article can be found online at https://doi.org/10.1556/004.2020.00019. 